\title{
DESENVOLVIMENTO DE PROCEDIMENTO DINÂMICO DE GERENCIAMENTO DE RISCOS DE PROJETO PARA APOIO À DECISÃO NA INDÚSTRIA DE ÓLEO E GÁS
}

\author{
Fábio Henrique de Souza \\ Universidade Federal Fluminense (UFF) \\ henrique.souza@ufrgs.br \\ Gilson Brito Alves Lima \\ Universidade Federal Fluminense (UFF) \\ Rua Passo da Pátria, 156, Bloco D, São Domingos, Niterói-RJ, CEP 24.220-240 \\ glima@id.uff.br \\ Luiz Octávio Gavião \\ Escola Superior de Guerra (ESG) \\ Fortaleza de São João - Av. João Luiz Alves, s/nº, Urca, Rio de Janeiro-RJ, CEP 22291-090 \\ luiz.gaviao67@gmail.com \\ Annibal Parracho Sant'Anna \\ Universidade Federal Fluminense (UFF) \\ Rua Passo da Pátria, 156, Bloco D, São Domingos, Niterói-RJ, CEP 24.220-240 \\ annibal.parracho@gmail.com
}

\section{RESUMO}

Com o aumento da competitividade no mercado na indústria de óleo e gás, cada vez mais tem sido necessário o controle de parâmetros que influenciam nos objetivos do projeto. $\mathrm{O}$ gerenciamento de riscos se torna essencial nesse quesito. Contudo, ainda há muito espaço para aprimoramento dos métodos usualmente aplicados. O objetivo desse artigo é a proposição de um procedimento integrado com base na FMEA (Failure Modes and Effects Analysis), em que a identificação e a priorização de riscos recebem tratamento próprio. A aplicação desse procedimento no estudo de caso de um projeto de manutenção de tanques em plataformas petrolíferas, através do uso de pesquisa de levantamento com a técnica de questionários, testou a pertinência e aplicabilidade do método.

Palavra-chave: Gerenciamento de riscos em projetos; Apoio à decisão; FMEA.

\begin{abstract}
With increasing market competitiveness in the oil and gas industry, it has been increasingly necessary to control parameters that influence project objectives. Risk management becomes essential in this regard. Nevertheless, there is still much room for improvement of the methods usually applied. The purpose of this article is the proposition of an integrated procedure based on FMEA (Failure Modes and Effects Analysis), in which the identification and priorization of risks receive their own treatment. The use of this procedure
\end{abstract}


in the case study of a tank maintenance project on oil platforms, through the use of survey research with the technique of questionnaries, tested the pertinence and applicability of the method.

Keywords: Project risk management; Decision support; FMEA.

\section{Como Citar:}

SOUZA, Fábio Henrique de; LIMA, Gilson Brito Alves; GAVIÃO, Luiz Octávio; SANT'ANNA, Annibal Parracho. Desenvolvimento de Procedimento Dinâmico de Gerenciamento de Riscos de Projeto para Apoio à Decisão na Indústria de Óleo e Gás. In: SIMPÓSIO DE PESQUISA OPERACIONAL E LOGÍSTICA DA MARINHA, 19., 2019, Rio de Janeiro, RJ. Anais [...]. Rio de Janeiro: Centro de Análises de Sistemas Navais, 2019.

\section{INTRODUÇÃO}

O objetivo deste estudo foi o desenvolvimento de um procedimento integrado de gerenciamento de riscos, utilizando-se, como base, a identificação de riscos na literatura e lições aprendidas de projetos complexos semelhantes, os quais foram analisados por especialistas de diversos setores para classificação dos riscos. Os diferentes tratamentos para cada risco foram priorizados e monitorados utilizando-se a técnica FMEA (Failure Modes and Effects Analysis). Utilizou-se um caso de uso através de pesquisa exploratória com grupo focal para verificar a adequação do instrumento proposto para um projeto de manutenção de tanques em plataformas de petróleo. O estudo limitou-se à etapa de iniciação do projeto, não abrangendo a reavaliação da FMEA após determinado tempo de continuidade dos serviços, o que ficou como tema de trabalhos futuros. Além disso, foram considerados somente riscos com aspectos negativos, ou seja, oportunidades (riscos positivos) não foram contempladas na análise.

O artigo abordou a proposição de um procedimento de gerenciamento de riscos, com uso da técnica FMEA, como ferramenta de apoio à decisão no que diz respeito ao tratamento de riscos para organizações. Sendo assim, a pesquisa tratou, por premissa, dos seguintes objetivos específicos: (1) modelar um instrumento de gerenciamento de riscos aplicado a uma organização com uso de questionários; (2) verificar se o grupo focal respondente estava adequado a parâmetros pré-estabelecidos para veracidade das respostas; (3) disponibilizar o instrumento de gerenciamento de riscos como um padrão que poderá ser seguido pelas organizações, seja utilizando-se as diretrizes de forma integral, seja de forma parcial.

O artigo foi dividido em três partes: na primeira etapa, foi realizada contextualização teórica com intuito de sintetizar o entendimento sobre gerenciamento de riscos e o que a literatura abordava no que diz respeito à aplicabilidade da técnica FMEA para gerenciamento de riscos. Na etapa seguinte, foi discutida a metodologia proposta aplicada a um projeto específico, no qual questionários foram aplicados a um grupo focal. Na terceira e última etapa foram discutidos os resultados obtidos com a pesquisa.

\section{CONTEXTUALIZAÇÃO TEÓRICA}

Em função da dinâmica global competitiva da indústria de óleo e gás, os riscos associados a projetos de manutenção vêm se tornando, cada vez mais, importantes de serem gerenciados. Porém, ainda há muito a se desenvolver com relação à maturidade de gerenciamento de riscos de pequenas a grandes empresas no Brasil. Meirelles (2018) menciona que, no Brasil, o gerenciamento de risco corporativo é pouco difundido na cultura empresarial, sendo mais utilizado nas grandes empresas, nacionais ou multinacionais. 


\subsection{Gerenciamento de Riscos em Projetos de Manutenção na Indústria de Óleo e GÁS}

O gerenciamento de riscos aplicado à manutenção na indústria de óleo e gás, por vezes, está associado a equipamentos ou processos específicos dentro de uma unidade, planta, refinaria, terminal ou plataforma petrolífera. Simon (2014), por exemplo, propõe uma análise de riscos associados ao armazenamento de óleo e gás em tanques. Jaderi, Ibrahim e Zahiri (2019) elaboraram um estudo de riscos associados à manutenção na falha de plantas petroquímicas.

PMI (2017) estabelece os seguintes processos de gerenciamento de riscos, conforme Figura 1.

Figura 1: Fluxo de gerenciamento de riscos

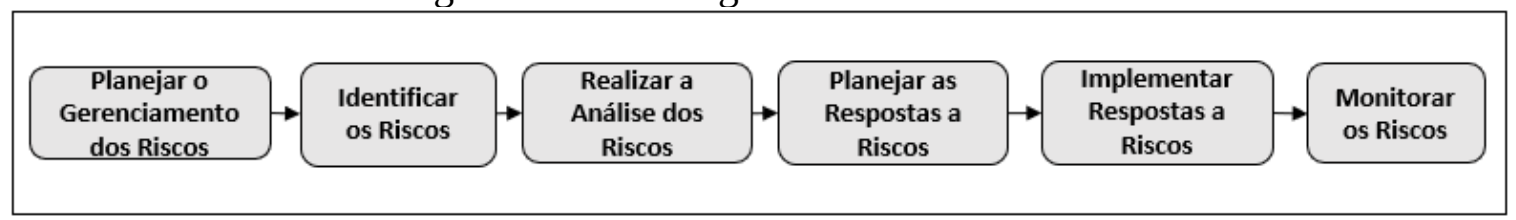

Fonte: Adaptado de PMI (2017)

A necessidade de identificação de riscos de um modo sistemático e adequado se torna, cada vez mais, importante no gerenciamento de projetos conforme aumentam as suas complexidades, tal como conclui Li, Zhang e Fu (2013), em seu estudo de metodologia de identificação de riscos para um projeto complexo de construção de uma ponte. Além disso, a análise eficaz, assim como o devido controle desses riscos e as ações atribuídas para tratamento dos mesmos, é primordial para que os projetos sejam finalizados com sucesso.

\subsubsection{Métodos de Identificação de Riscos}

Os riscos podem ser segmentados em dois graus: risco individual do projeto e risco geral do projeto. O risco individual do projeto é um evento ou condição incerta que, se ocorrer, provocará um efeito positivo ou negativo no projeto. Já o risco geral do projeto é o efeito da incerteza do projeto no seu todo, decorrente de todas as fontes de incerteza, incluindo riscos individuais, representando a exposição das partes interessadas às implicações de variações no resultado do projeto, sejam positivas ou negativas (PMI, 2017).

A partir dessa definição, pode-se compor o risco geral do projeto a partir de um conjunto de riscos individuais. Para tanto, há a necessidade de identificarem-se os riscos. Um diagrama de métodos de identificação de riscos pode ser visto na Figura 2:

Figura 2: Diagrama de métodos de identificação de riscos

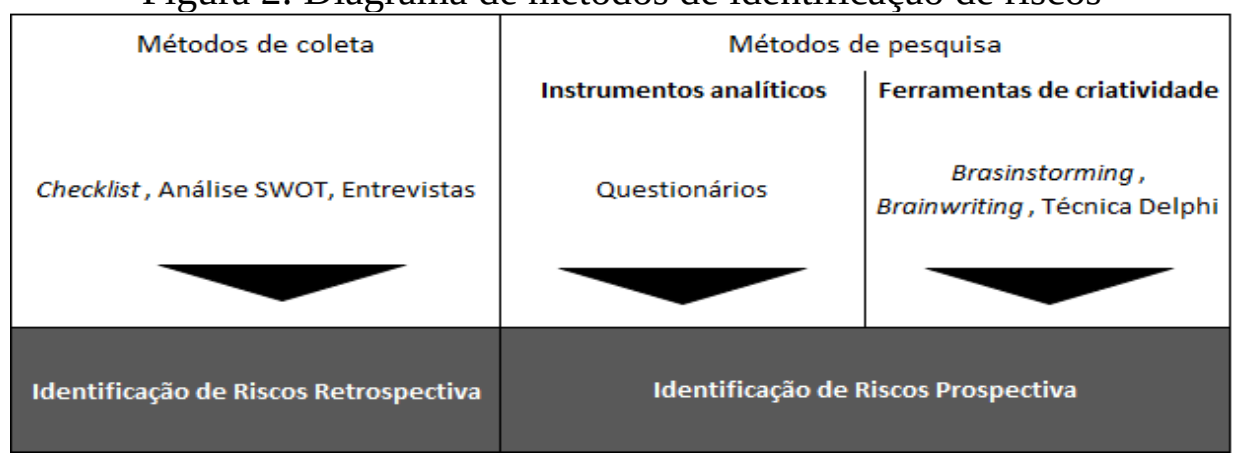

Fonte: Adaptado de Baumann (2016) 
O checklist é um método de coleta amplamente utilizado que se desdobra de várias maneiras, como, por exemplo, na estrutura analítica de riscos (EAR), de modo a se ter riscos associados a determinadas áreas. Li, Zhang e Fu (2013) propõem uma WBS-RBS (Work Breakdown Structure - Risk Breakdown Structure), também conhecida como EAP-EAR (Estrutura Analítica de Projeto - Estrutura Analítica de Riscos) para o projeto de construção de uma ponte de grande porte, como uma forma de facilitar o processo de identificação dos riscos de forma mais completa e sistemática. Souza e Barcaui (2014, p. 12) mencionam que:

\begin{abstract}
A identificação de riscos se torna mais metódica e facilitada com o desenvolvimento de uma Estrutura Analítica de Riscos. Entretanto, deve-se ter o cuidado de estabelecer categorias que auxiliem na identificação de novos riscos e não categorias que limitem os tipos de riscos que possam surgir no projeto.
\end{abstract}

A análise SWOT (Strengths, Weakness, Opportunities and Threats) permite identificar forças (strengths), fraquezas (weakness), oportunidades (opportunities) e ameaças (threats) de um determinado projeto ou área em que se levantem os riscos. Jiang et al. (2018) utilizam este recurso para analisar centenas de artigos e documentos do governo chinês para identificação de riscos e oportunidades em projetos da indústria de construção na China.

Quanto às entrevistas, segundo Gray (2012, p. 299), “uma entrevista é uma conversa entre pessoas, na qual uma cumpre o papel de pesquisador”. Chan et al. (2014) utilizam entrevistas para a identificação de riscos em projetos no setor de abastecimento de água na China.

Questionários podem ter perguntas abertas ou fechadas, sendo que, no primeiro caso, a principal vantagem estaria relacionada à possibilidade de riqueza de informações e, no segundo, ao fato de facilitar o processamento e análise das informações, em função de gerar respostas padronizadas (GRAY, 2012). Wu et al. (2017), por exemplo, utilizam questionários para levantamento de fatores de riscos em projetos na área de geração de energia através de palha na China.

Há, também, ferramentas que promovem o uso da criatividade, como o Brainstorming e o Brainwriting, em que cada componente do grupo lança suas ideias de riscos, a primeira em reunião e a segunda por proposições escritas. Gray (2012) menciona que o Brainstorming é uma técnica conhecida de solução de problemas para gerar e refinar ideias.

Na técnica Delphi, através de um grupo de participantes, busca-se o consenso entre os mesmos, anonimamente, quanto aos riscos levantados para o projeto. Por intermédio de um componente do grupo, busca-se o alinhamento sobre quais riscos devem ser considerados no levantamento realizado, passando-se o conjunto de riscos levantados pelos envolvidos para comentários adicionais com uso de questionários padrões sobre o que se avalia. Com várias rodadas, chega-se à conclusão de quais serão considerados. Xu et al. (2010) utilizam a técnica Delphi para apoio à identificação de riscos adicionais para projetos de infraestrutura na China.

\title{
2.1.2. Etapa de Análise de Riscos
}

Após a etapa de identificação dos riscos, faz-se necessária a análise dos mesmos. Segundo PMI (2017), primeiro, se realiza a análise qualitativa dos riscos, na qual são priorizados os riscos do projeto. Em um segundo momento, realiza-se a análise quantitativa, na qual se analisa numericamente o efeito combinado dos riscos identificados e outras fontes de incerteza nos objetivos gerais do projeto, de modo que se consiga quantificar a exposição ao risco geral do projeto. No quesito de priorização para tratamento, OGC (2011) menciona 
a técnica da análise de Pareto, para concentrar esforços no gerenciamento de riscos que têm maior potencial de impactar nos objetivos do projeto.

PMI (2017) sugere, mas não limita, o uso de entrevistas para realização da análise qualitativa. Nesse caso, a coleta de dados pode ser realizada através de entrevistas estruturadas ou semiestruturadas. Gray (2012) menciona que, quando for necessário o uso de perguntas padronizadas, o questionário torna-se ideal e permite, se necessário, uma abordagem analítica explorando as relações entre variáveis.

\subsubsection{Uso da Técnica FMEA}

O próximo passo é o planejamento de respostas aos riscos e a implementação das ações de respostas com o posterior monitoramento dos riscos. A técnica de Análise dos Modos e Efeitos de Falhas, também conhecida como FMEA, segundo Palady (1997), "é uma ferramenta para prognóstico de problemas, é um procedimento para desenvolvimento e execução de projetos, processos ou serviços, novos ou revisados e é o diário de um projeto, processo ou serviço”. Pode ser aplicada a projetos levando em consideração a análise, priorização, planejamento de respostas e até mesmo o monitoramento dos riscos. Reis (2015) aplica a técnica FMEA para análise de riscos para um estudo de caso específico no ramo de manutenção. Mangla, Luthra e Jakhar (2017) utilizam a ferramenta FMEA integrada com lógica Fuzzy para análise de riscos para um estudo de caso na área de Green Supply Chain na Índia.

\subsection{CONFIABILIDADE DE QUESTIONÁRIOS}

A confiabilidade de questionários, segundo Gray (2012), está associada à constância nos resultados e o nível dessa constância pode ser obtido através de um coeficiente de confiabilidade, como por exemplo o coeficiente alfa de Cronbach. O alfa de Cronbach é obtido através da fórmula exposta na Figura 3, a seguir:

Figura 3: Fórmula do cálculo do coeficiente alfa de Cronbach

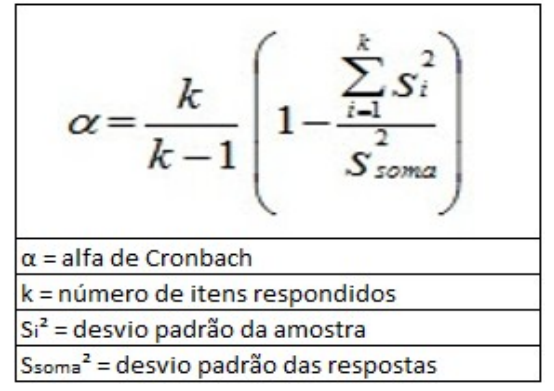

Fonte: Adaptado de Cronbach (1951)

Hora et al. (2010) aplicam o coeficiente alfa de Cronbach em um estudo para análise da confiabilidade de questionários para avaliação da qualidade de serviços prestados. Como resultado, foram removidos alguns itens do questionário, aumentando a sua confiabilidade.

\section{ABORDAGEM METODOLÓGICA}

A metodologia proposta baseou-se em pesquisa exploratória de campo com grupo focal, referente a um projeto específico de uma organização que presta serviços de manutenção, construção e montagem. Como procedimento metodológico, foi realizada a aplicação de questionários contemplando riscos previamente levantados na literatura em conjunto com riscos adicionais obtidos de lições aprendidas de projetos semelhantes. Os 
questionários contemplavam colunas para classificação dos riscos quanto à severidade, probabilidade e dificuldade de detecção, com base na técnica FMEA.

Para análise da consistência do questionário, foi aplicado um teste piloto inicialmente submetido a dois respondentes. Após preenchimento das respostas, o questionário sofreu os ajustes necessários, sendo, na sequência, reaplicado à amostra selecionada correspondente ao grupo focal.

Essa amostra se caracterizou como sendo não probabilística por conveniência, composta de grupo focal de catorze profissionais atuantes na área de gerenciamento de projetos e/ou envolvidos diretamente nas atividades do projeto analisado. A confiabilidade do questionário aplicado foi analisada através do uso do coeficiente de alfa de Cronbach, aplicado para cada seção das respostas referentes à severidade, probabilidade e detectabilidade.

O questionário foi entregue em mídia impressa aos respondentes, tendo sido aplicado ao longo de um período de uma quinzena. A aplicação foi supervisionada para nivelamento de eventuais dúvidas dos respondentes.

O teste do procedimento integrado foi realizado através da aplicação de todo o processo em um grupo focal para um projeto de manutenção de tanques em plataformas petrolíferas, abrangendo o período de iniciação e mobilização do projeto, considerando riscos negativos identificados.

Os riscos identificados foram analisados quanto à severidade, probabilidade e dificuldade de detecção. A classificação desses foi realizada através do produto de probabilidade pela severidade. Foram selecionados 20\% dos riscos considerados mais críticos, o que representaria $80 \%$ do efeito sobre os objetivos do projeto. Esses riscos foram selecionados para tratamento de resposta a fim de estipular-se as ações e responsáveis por implementá-las.

As análises das informações obtidas na pesquisa foram realizadas com o uso do software Power BI, da Microsoft. Esse programa foi escolhido pelo fato de o mesmo apresentar informações gerenciais de forma gráfica com maior facilidade do que outros softwares tradicionais, além de facilitar a própria apresentação de dados e filtros considerando a tecnologia de Business Intelligence.

\subsection{Proposição do Procedimento InTegrado Para Gerenciamento de Risco}

Um procedimento integrado para gerenciamento de riscos foi proposto incorporando métodos de identificação de riscos com base na literatura existente, análise dos riscos com uso de questionários e uma matriz já considerando aplicação da ferramenta FMEA. Por fim, as respostas e o monitoramento dos riscos com uso da FMEA ficam para um estudo futuro, assim como a reaplicação da FMEA para acionar-se a fase de monitoramento.

O processo de aplicação da FMEA adaptado ao gerenciamento de riscos foi realizado através do levantamento das respostas dos questionários preenchidos, dos quais foi possível obter os resultados referentes aos índices de severidade, probabilidade e dificuldade de detecção para cada risco. No entanto, a avaliação dos resultados ocorreu após a análise dos mesmos, priorizando-se com a técnica Pareto em função do produto da severidade e da probabilidade, no que diz respeito a quais deveriam ser tratados. Para a ordem de implementação das ações, entrou a questão da dificuldade de detecção dos riscos, pois riscos de difícil detecção devem ser prioritários em relação a riscos mais facilmente perceptíveis, dado o fato de que se pode estar sofrendo consequências de um risco que já virou fato e ainda não se ter percebido a sua ocorrência. Palady (1997) menciona que para a realização da FMEA devem ser considerados o índice de ocorrência, o índice de severidade, o índice de detecção e o índice de risco ou grau de prioridade de risco (Risk Priority Number - RPN), sendo este último o produto dos três anteriores. 
Dados esses indicadores, a planilha para avaliação e determinação das respostas aos riscos com base na FMEA adaptada a riscos pode ser visualizada na Figura 4, a seguir.

Figura 4: Planilha de avaliação e determinação das respostas aos riscos com base na FMEA

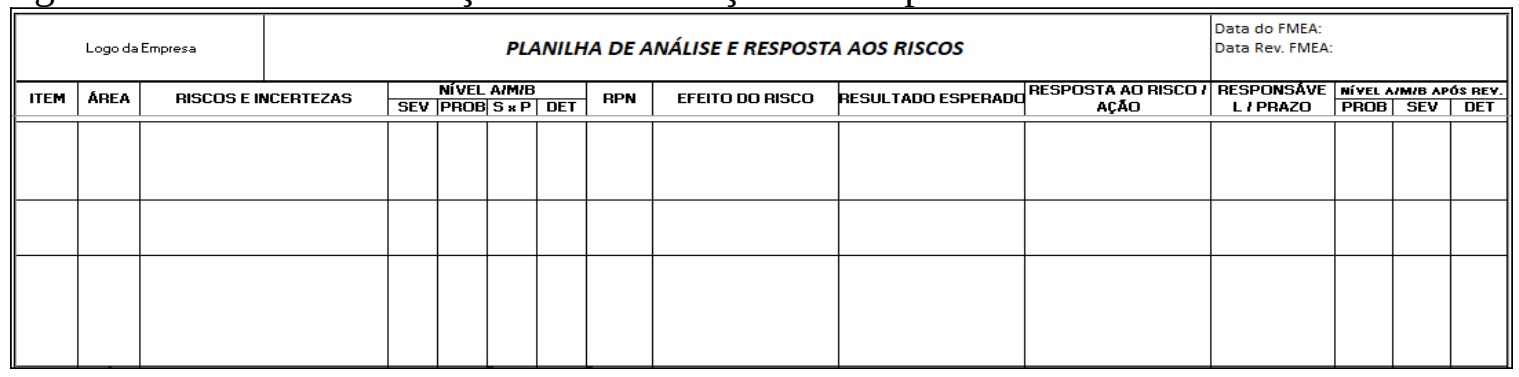

Fonte: Adaptado de Reis (2015)

Basicamente, a execução do procedimento integrado seguiu o fluxograma conforme disposto na Figura 5, a seguir.

Figura 5: Fluxograma do procedimento integrado com FMEA

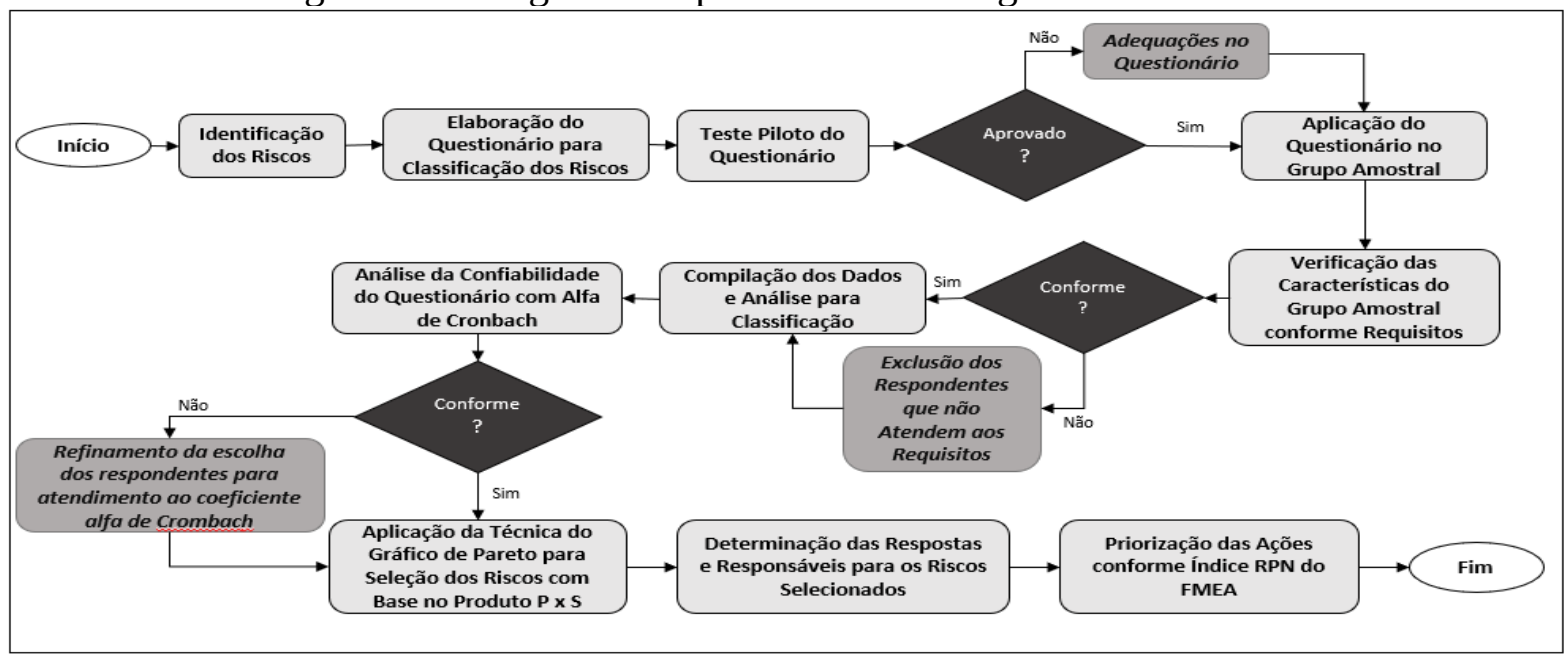

Fonte: Elaboração própria

Nota-se que o fluxograma foi elaborado até a etapa de priorização das ações, não abrangendo as etapas posteriores de aplicação das ações e monitoramento dos riscos.

\subsection{IDENTIFICAÇÃo dOS Riscos PARA O GRUPO FoCAL}

Como se trata de pesquisa exploratória com grupo focal, o planejamento do gerenciamento de riscos ficou atrelado a fontes de dados existentes ou a serem levantadas de acordo com o projeto estudado. Nesse quesito, decidiu-se que a identificação dos riscos seria realizada com base na literatura já existente, somada a listas de verificação de projetos semelhantes dentro da empresa, ou seja, lições aprendidas. Oliveira e Lima (2015) identificam riscos em projetos complexos, o que se assemelha em vários aspectos a projetos de manutenção na indústria petroquímica, objeto da pesquisa em questão. Além disso, o autor realiza a identificação de riscos utilizando uma EAR básica que considera as áreas de conhecimento de gerenciamento de projetos segundo o PMI (Project Management Institute), porém com uma versão mais antiga do PMBOK, não considerando a nona área de conhecimento, gerenciamento das partes interessadas. De acordo com o PMI (2017), as áreas de conhecimento em gerenciamento de projetos estão dispostas conforme Figura 6, a seguir, 
com a nomenclatura utilizada para o processo de identificação de riscos no estudo em questão:

Figura 6: Áreas de conhecimento de projetos e nomenclatura utilizada no processo de identificação de riscos

\begin{tabular}{|c|c|}
\hline Áreas de Conhecimento de Acordo com o PMBOK 6a Edição (2017) & Nomenclatura Utilizada na Identificação dos Riscos \\
\hline 1 Gerenciamento da Integração do Projeto & Integração \\
\hline 2 Gerenciamento do Escopo do Projeto & Escopo \\
\hline 3 Gerenciamento do Cronograma do Projeto & Tempo \\
\hline 4 Gerenciamento dos Custos do Projeto & Custos \\
\hline 5 Gerenciamento da Qualidade do Projeto & Qualidade \\
\hline 6 Gerenciamento dos Recursos do Projeto & $\mathrm{RH}$ \\
\hline 7 Gerenciamento das Comunicações do Projeto & Comunicação \\
\hline 8 Gerenciamento dos Riscos do Projeto & $*$ \\
\hline 9 Gerenciamento das Aquisições do Projeto & Aquisiçôes \\
\hline 10 Gerenciamento das Partes Interessadas do Projeto & Partes Interessadas \\
\hline
\end{tabular}

Fonte: Elaboração própria baseada em PMI (2017)

Sendo assim, foram utilizados os riscos levantados, adequando-se a descrição dos mesmos ao projeto do estudo. Adicionalmente, contemplou-se a área de gerenciamento das partes interessadas e foram acrescentados riscos identificados de projetos iniciados anteriormente na empresa, diferentes dos já levantados.

\subsection{Processo de Análise, AÇões e Monitoramento dos Riscos}

Para evitar o viés sobre o entendimento da classificação de cada risco, foi realizada análise através de questionários distribuídos para profissionais da empresa com determinados critérios. Segundo Gray (2012), ao se aplicar questionários e ao se elaborar as perguntas, deve-se certificar-se que o grupo respondente escolhido tenha conhecimento para responder ao questionário. Logo, uma das seções do questionário para o grupo focal coletou informações sobre características dos respondentes para verificar a validade da aplicação do questionário, além de ter sido realizada a análise da confiabilidade dos questionários por meio do uso do coeficiente Alfa de Cronbach. Outra seção considerada no questionário foi a avaliação, por parte dos respondentes, de quais áreas de conhecimento em gerenciamento de projetos têm maior influência na gestão de riscos, de modo a poder-se avaliar se o resultado da pesquisa como um todo está em alinhamento com o pensamento comum no que se refere aos segmentos das áreas de conhecimento que mais influenciam em riscos.

Por fim, foi desenvolvida a seção principal do questionário, na qual se considerou a listagem dos riscos levantados para classificação dos respondentes. Nesse quesito, considerou-se a avaliação através da matriz de probabilidade e severidade, adicionando-se uma coluna de dificuldade de detecção do risco, com intuito de posterior uso na FMEA. Não obstante, a classificação dos riscos para verificar-se quais seriam tratados está baseada apenas na matriz de probabilidade e severidade. Cayanan, Navarro e Wagner (2013) aplicaram a matriz de probabilidade e severidade para quantificação do risco de falha de um estudo de caso de um equipamento elétrico e determinam, com sucesso, a prioridade de reparo ou substituição de peças do equipamento, reduzindo, assim, o risco de falha.

A classificação dos riscos foi baseada na escala Likert, conforme abordado por Gray (2012), usual para perguntas de escala ou classificação, variando discretamente de um até cinco. Na Figura 7, a seguir, é demonstrado um exemplo de pergunta do questionário a ser respondido, referente a um "Risco X", circulando-se as numerações correspondentes à classificação: 
Figura 7: Exemplo de pergunta do questionário com escala Likert

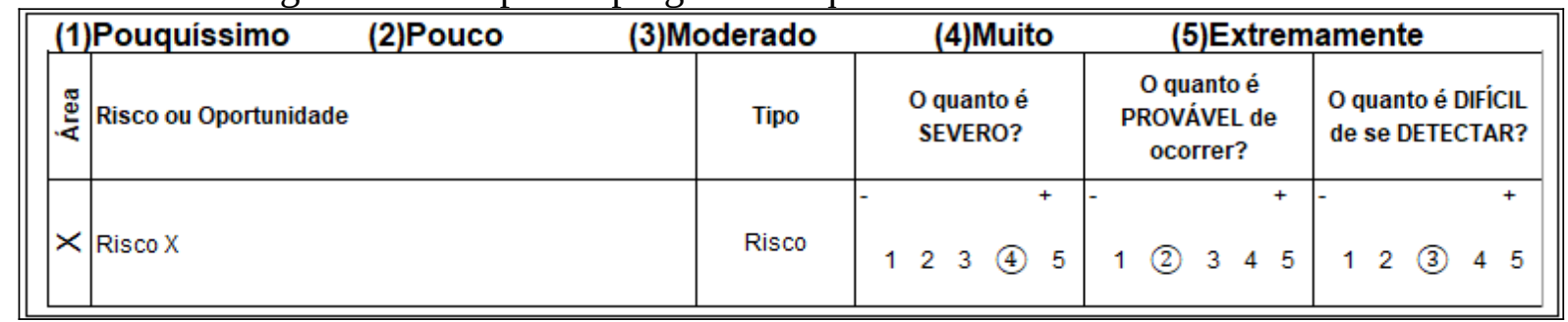

Fonte: Elaboração própria baseada em Gray (2012)

Após a classificação dos riscos, se determinou, com base na curva de Pareto, quais riscos serão priorizados para tratamento. Consideraram-se, com base na consulta a especialistas do grupo focal, que deveriam ser tratados os riscos que compõem a margem acumulada acima de $80 \%$ de efeito sobre os objetivos do projeto, sendo o efeito o produto da probabilidade pela severidade.

Assim, entende-se que $20 \%$ dos riscos têm consequência de $80 \%$ do efeito nos objetivos do projeto e, portanto, foram priorizados para tratamento. Para exemplificar, na Figura 8, a seguir, considerar-se-ia que somente os riscos “11", “12" e "13" seriam priorizados devido ao fato de os mesmos representarem $80 \%$ da intensidade do efeito sobre os objetivos do projeto. A classificação quanto à dificuldade de detecção foi utilizada para se priorizar quais ações devem ser implementadas prioritariamente, para os riscos selecionados como críticos.

Figura 8: Exemplo da análise do efeito dos riscos para consideração na curva de Pareto

\begin{tabular}{|c|c|c|c|c|c|c|}
\hline Riscos & $\begin{array}{c}\text { Severidade } x \\
\text { Probabilidade }\end{array}$ & $\begin{array}{c}\text { Porcentagem } \\
\text { Acumulada }\end{array}$ & Observação & \multirow{5}{*}{$\begin{array}{l}2500 \\
2000\end{array}$} & Pareto de Riscos Associados a um Projeto & \multirow{3}{*}{$\begin{array}{l}100 \% \\
90 \%\end{array}$} \\
\hline Risco 1 & 1150 & $5 \%$ & \multirow{10}{*}{$\begin{array}{l}\text { Não considerado } \\
\text { para tratamento }\end{array}$} & & & \\
\hline Risco 2 & 1200 & $10 \%$ & & & & \\
\hline Risco 3 & 1350 & $16 \%$ & & & & $80 \%$ \\
\hline Risco 4 & 1400 & $22 \%$ & & & & $70 \%$ \\
\hline Risco 5 & 1600 & $29 \%$ & & & & $60 \%$ \\
\hline Risco 6 & 1650 & $37 \%$ & & & & $50 \%$ \\
\hline Risco 7 & 1700 & $44 \%$ & & \multirow[t]{2}{*}{1000} & & $40 \%$ \\
\hline Risco 8 & 1750 & $52 \%$ & & & & $30 \%$ \\
\hline Risco 9 & 1950 & $60 \%$ & & \multirow[t]{2}{*}{500} & & $20 \%$ \\
\hline Risco 10 & 2100 & $70 \%$ & & & & $10 \%$ \\
\hline Risco 11 & 2250 & $80 \%$ & \multirow{3}{*}{$\begin{array}{l}\text { Considerados para } \\
\text { tratamento }\end{array}$} & & & \\
\hline Risco 12 & 2300 & $90 \%$ & & & & \\
\hline Risco 13 & 2350 & $100 \%$ & & & & \\
\hline
\end{tabular}

Fonte: Elaboração própria

Cabe destacar que a empresa do estudo adota, para as questões de probabilidade, severidade e dificuldade de detecção, a própria classificação do efeito dos riscos (produto da probabilidade com a severidade) sobre os objetivos do projeto. Essa classificação é caracterizada por ser uma divisão linear, em três partes, da amplitude dos resultados das classificações para cada uma das características, tal como pode ser visualizado na Figura 9.

Figura 9: Padronização da classificação dos riscos 


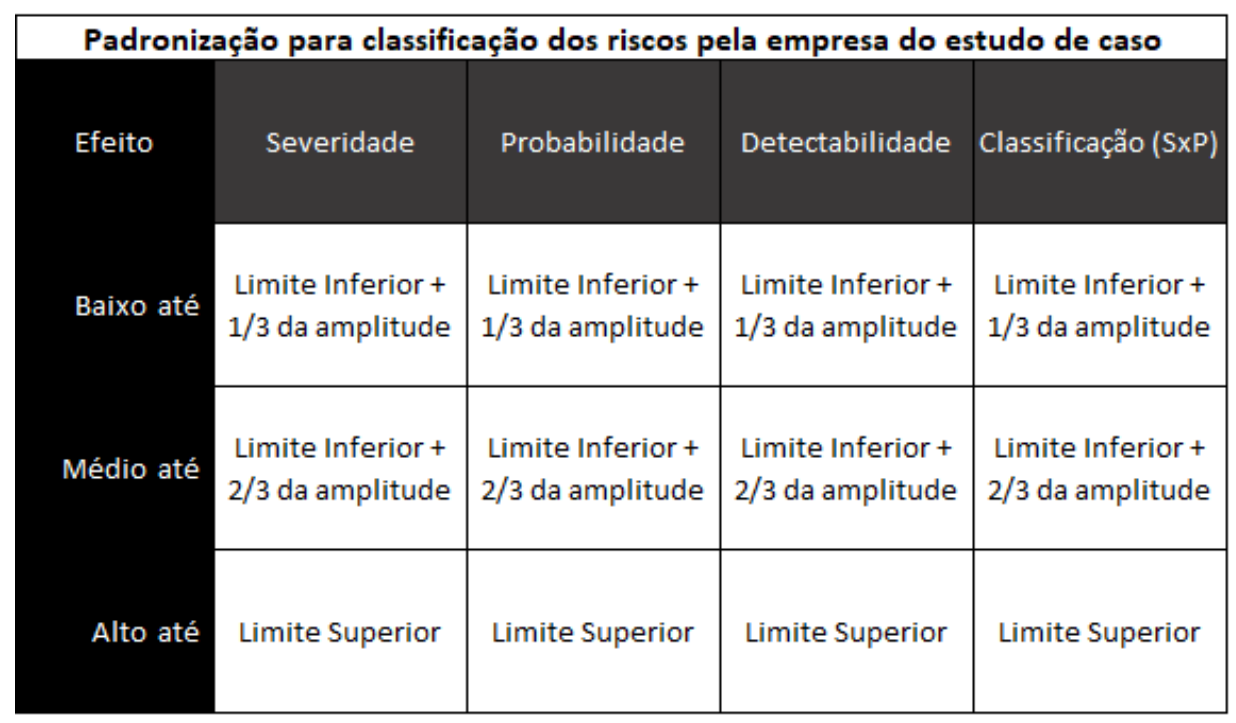

Fonte: Elaboração própria baseada na empresa estudada

Tendo sido a FMEA aplicada e já determinadas as respostas aos riscos, implementam-se as ações. O monitoramento deverá ser realizado semestralmente, conforme determinação da empresa, quando se verificará a eficácia das ações implementadas e qual a nova classificação que se dará aos riscos conforme efeito das respostas acionadas. Esse processo é realizado por meio da reaplicação da $F M E A$, através de uma nova rodada de questionários e seu processo subsequente (com possibilidade de inserção de novos riscos, conforme alteração de prioridade). Essa última etapa de reaplicação da $F M E A$ ficará para estudos futuros, não sendo objeto desse artigo.

\section{RESULTADOS DA PESQUISA}

$\mathrm{Na}$ fase de identificação dos riscos, no total, foram considerados 110 riscos distribuídos conforme Figura 10.

Figura 10: Quantidade e proporção de riscos levantados para cada área de conhecimento

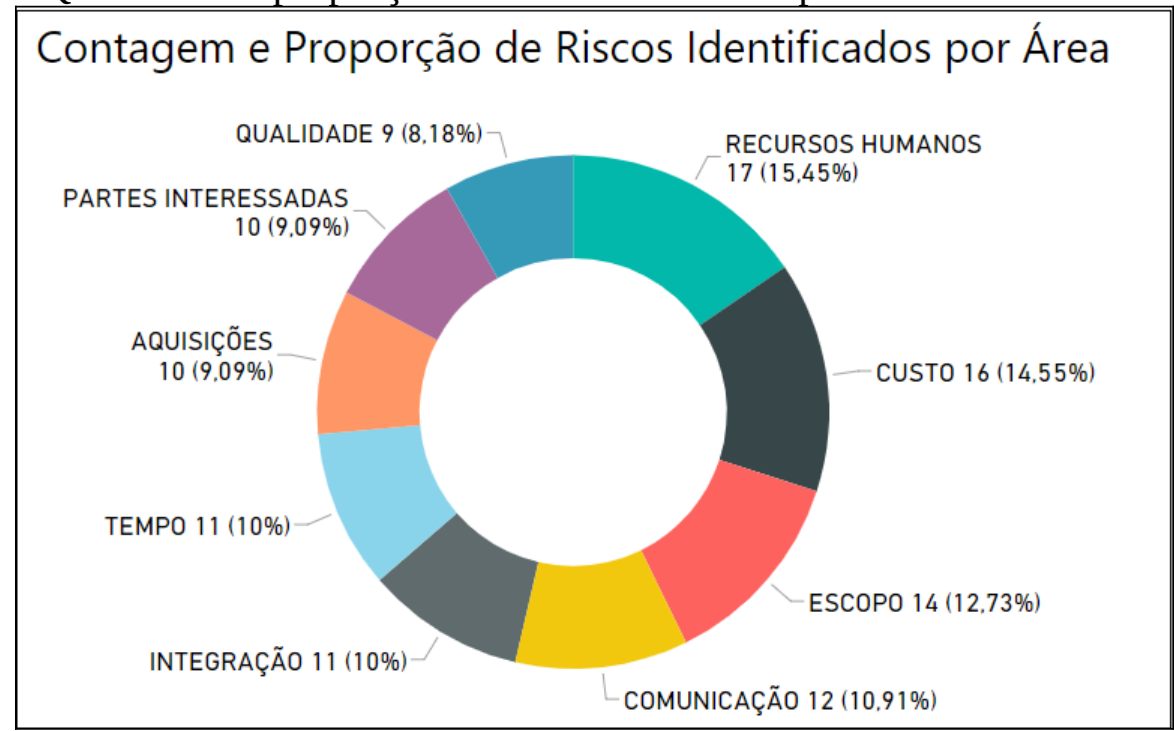

Fonte: Elaboração própria

Com relação à característica dos respondentes do questionário, na Figura 11, de catorze respondentes, pode ser visualizada a proporção por setor. 
Figura 11: Proporção de respondentes por setor da empresa

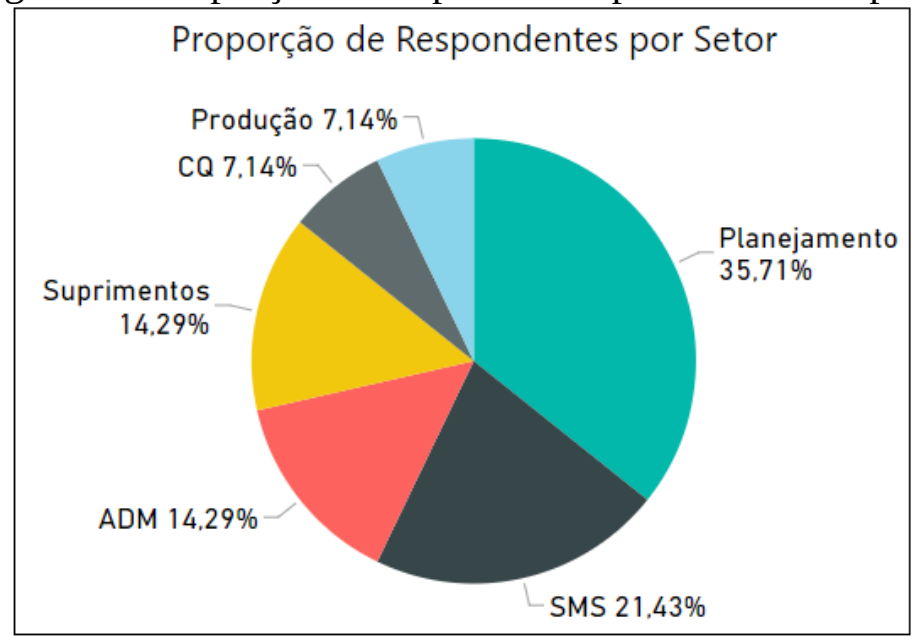

Fonte: Elaboração própria

Logo, pode-se considerar a quantidade de respondentes por setor bem distribuída, visto que, proporcionalmente, o setor de planejamento é o que possui maior quantidade de funcionários na empresa - desconsiderando-se mão de obra direta, que, em sua maioria, não teria conhecimento adequado sobre o assunto para responder ao questionário.

Quanto à análise da percepção de risco por área de conhecimento, o resultado pode ser visualizado na Figura 12.

Figura 12: Percepção de risco por área de conhecimento

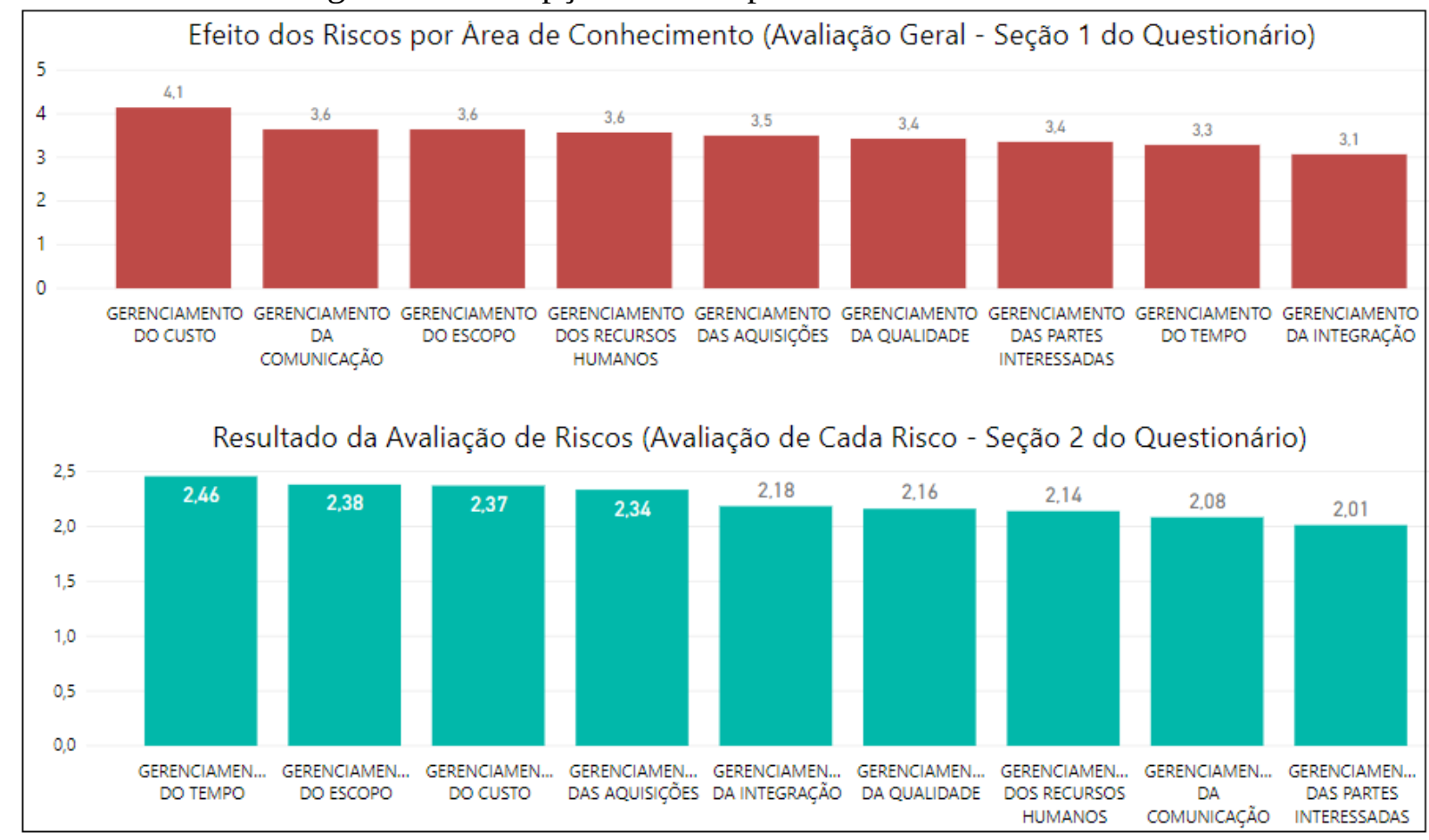

Fonte: Elaboração própria

Para as primeiras cinco áreas classificadas como de maior efeito em relação a riscos, três estão presentes simultaneamente nos resultados da classificação das áreas em 
relação à classificação da média dos riscos por área (custo, escopo e aquisições), o que foi considerado dentro do esperado pelos especialistas da empresa.

Quanto à confiabilidade dos questionários, foram calculados os coeficientes alfa de Cronbach para cada tipo de classificação (severidade, probabilidade e detectabilidade). $\mathrm{O}$ resultado pode ser visualizado na Figura 13, a seguir.

Figura 13: Resultados do cálculo dos coeficientes alfa de Cronbah

\begin{tabular}{|c|c|r|}
\hline \multicolumn{3}{|c|}{ Resultados de Alfa de Cronbach } \\
\hline $\mathrm{S}$ & $\alpha$ severidade & 0,984 \\
\hline $\mathrm{P}$ & $\alpha$ probabilidade & 0,979 \\
\hline $\mathrm{D}$ & $\alpha$ detectabilidade & 0,990 \\
\hline
\end{tabular}

Fonte: Elaboração própria

Sendo assim, como nos três coeficientes o resultado foi maior que 0,7 , entende-se que o questionário possui uma confiabilidade aceitável. Logo, não houve necessidade de adequar-se o questionário ou a seleção de dados que seriam considerados.

Quanto à análise e classificação dos riscos, com os resultados obtidos dos questionários respondidos, os intervalos que determinavam o efeito das características dos riscos ficaram conforme a Figura 14.

Figura 14: Padronização dos intervalos de acordo com os resultados obtidos nos questionários

\begin{tabular}{|c|l|c|c|c|c|}
\hline \multicolumn{5}{|c|}{ Padronização para classificação dos riscos pela empresa do estudo de caso } \\
\hline \multicolumn{2}{|c|}{ Efeito } & Severidade & Probabilidade & Detectabilidade & Classificação (SxP) \\
\hline \multirow{2}{*}{ Baixo } & de & 1,000 & 1,000 & 1,000 & 1,000 \\
\cline { 2 - 6 } & até & 3,213 & 2,594 & 2,332 & 9,565 \\
\hline \multirow{2}{*}{ Médio } & de & 3,214 & 2,595 & 2,333 & 9,566 \\
\cline { 2 - 6 } & até & 3,928 & 3,261 & 2,737 & 13,060 \\
\hline \multirow{2}{*}{ Alto } & de & 3,929 & 3,262 & 2,738 & 13,061 \\
\cline { 2 - 6 } & até & 5,000 & 5,000 & 5,000 & 25,000 \\
\hline
\end{tabular}

Fonte: Elaboração própria

A ordenação dos riscos de acordo com o seu efeito, referente ao produto da severidade e da probabilidade, serviu de base para se verificar a porcentagem acumulada desse produto para os riscos e se realizar a análise pelo gráfico de Pareto, conforme pode ser observado na Figura 15, a seguir.

Figura 15: Gráfico de Pareto para seleção dos riscos a serem tratados 


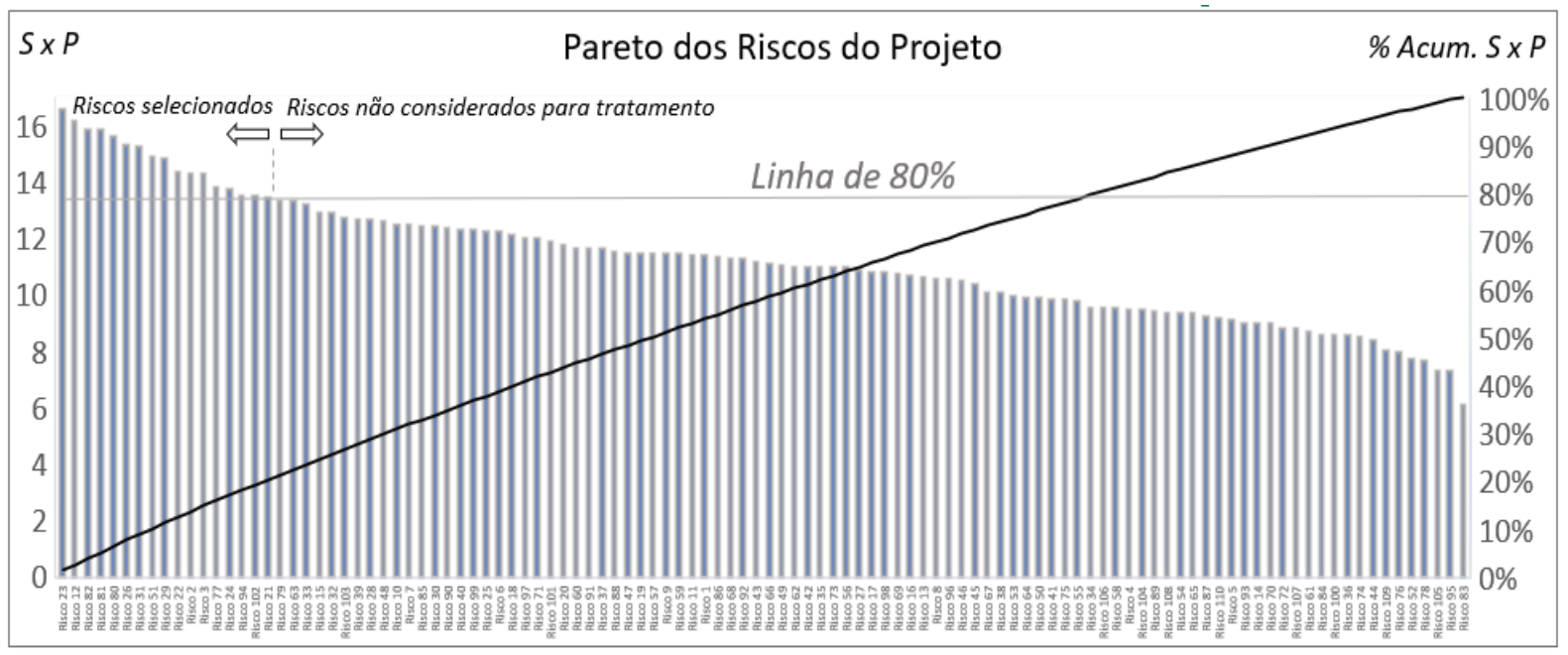

Fonte: Elaboração própria

Dessa forma, foram considerados os riscos que contemplam 20\% do valor total acumulado do efeito resultante do produto da probabilidade pela severidade, sendo que esses responderiam por $80 \%$ das consequências nos objetivos do projeto, pelo gráfico de Pareto. A representação da seleção dos riscos pode ser visualizada no gráfico de probabilidade e severidade conforme Figura 16, a seguir.

Figura 16: Gráfico de Severidade x Probabilidade dos Riscos do Estudo

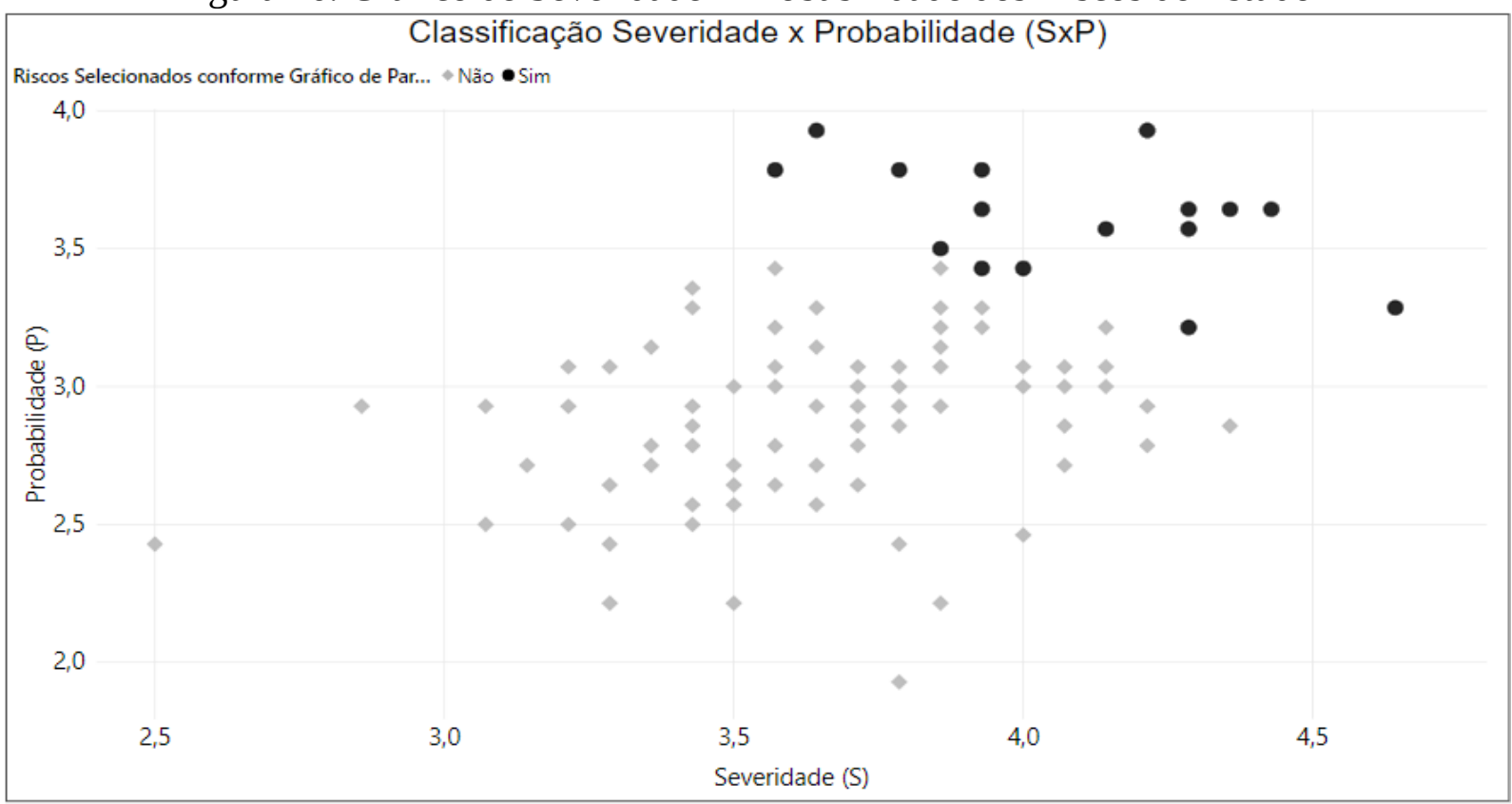

Fonte: Elaboração própria

Após selecionados os riscos, com o apoio de especialistas, inclusive membros do grupo focal respondente dos questionários de análise, foram determinados os efeitos da ocorrência do risco, qual seria o resultado esperado, a resposta ao risco com a ação a ser tomada e os responsáveis por essas ações. Assim, o procedimento integrado com a FMEA foi aplicado no estudo em questão, sendo determinadas ações específicas para tratamento de cada risco selecionado de acordo com os critérios discutidos. 


\section{CONCLUSÕES}

O uso do procedimento integrado com FMEA para gerenciamento de riscos na área de manutenção pôde contribuir no levantamento sistemático dos riscos, na análise desses minimizando a questão do viés com o uso de questionários e em um planejamento de respostas direcionado para os riscos de maior prioridade. $\mathrm{O}$ monitoramento dos riscos com posterior reavaliação da $F M E A$, fica como sugestão de desenvolvimento de trabalhos futuros.

Cabe destacar que esse processo é passível de se tornar padrão da empresa pesquisada, assim como para outras empresas que possam aplicá-lo. A ideia de utilizar técnicas amplamente difundidas no meio acadêmico para o meio das organizações contribui não só para as empresas que possam ter resultados melhorados quando utilizam técnicas com maior embasamento científico para gerenciamento de riscos, mas também para o meio acadêmico que comprova a utilidade da metodologia estudada. Outrossim, o uso dessas técnicas contribui para a evolução da maturidade de gerenciamento de risco nas organizações brasileiras.

Por fim, pôde-se concluir, por meio da aplicação prática do processo, que o procedimento integrado proposto é viável e pertinente, além de fornecer um desdobramento das etapas de gerenciamento de riscos que podem ainda derivar na integração de outras técnicas discutidas e até mesmo não discutidas nesse artigo.

\section{REFERÊNCIAS BIBLIOGRÁFICAS}

[1] BAUMANN, Susanne; ERBER, Iris; GATTRINGER, Magdalena. Selection of Risk Identification Instruments, ACRN Oxford Journal of Finance and Risk Perspectives. 5.2, p. 27-41, 2016.

[2] CAYANAN, Christopher; NAVARRO, Rey; WAGNER, Emie. A risk based approach for prioritizing electrical equipment replacement and repair. Record of Conference Papers - Annual Petroleum and Chemical Industry Conference, p. 1-8, 2013.

[3] CHAN, Albert P. C.; LAM, Patrick T. I.; WEN, Yang; AMEYAW, Ernest E.; WANG, Shouqing; KE, Youngjan. Cross-sectional analysis of critical risk factors for PPP water projects in China. Journal of Infrastructure Systems, 21(1), 2015.

[4] CRONBACH, J. L. Coefficient alpha and the internal structure of tests. V. 16. No. 3, pp. 297-334, Psychometrika, set. 1951.

[5] GRAY, David E. Pesquisa no mundo real. 2. Ed. Porto Alegre: Penso, 2012.

[6] HORA, Henrique R. M., MONTEIRO, Gina T. R., ARICA, Jose. Confiabilidade em questionários para qualidade: um estudo com coeficiente alfa de Cronbach. Periódico Produto e Produção, vol. 11, n. 2, p. 85 - 103, jun. 2010.

[7] JADERI, Fereshteh; IBRAHIM, Zelina Z.; ZAHIRI, Mohammad R. Criticality analysis of petrochemical assets using risk based maintenance and the fuzzy inference system. Process Safety and Environmental Protection, 121, p. 312-325, 2019.

[8] JIANG, Rui; MAO, Chao; HOU, Lei; WU, Chengke; TAN, Jiajuan. A SWOT analysis for promoting off-site construction under the backdrop of China's new urbanisation. Journal of Cleaner Production, 173, p. 225-234, 2018. 
[9] LI, Qing-Fu; ZHANG, Peng; FU, Yan-Chao. Risk identification for the construction phases of the large bridge based on WBS-RBS. Research Journal of Applied Sciences, Engineering and Technology, 6(9), 2013.

[10] MANGLA, Sachin K.; LUTHRA, Sunil., JAKHAR, S. Benchmarking the risk assessment in green supply chain using fuzzy approach to FMEA: insights from an Indian case study. Benchmarking: An International Journal. Vol. 25, 8, p. 2660-2687, 2017.

[11] MEIRELLES, Rogério. Gerenciamento de risco corporativo e avaliação da maturidade empresarial em gerenciamento de risco corporativo: um estudo de caso na Akzonobel. 2018. Dissertação (Mestrado Profissional em Empreendedorismo). FEA da Universidade de São Paulo - USP, São Paulo.

[12] OGC. Gerenciamento de projetos com PRINCE2. Londres: TSO, 2011.

[13] OLIVEIRA, Rodolpho A. M; LIMA, Gilson B. A. Fatores críticos de risco na perspectiva de projetos complexos em regime EPC Turnkey. 2015. Dissertação (Mestrado em Engenharia de Produção). Escola de Engenharia - Universidade Federal Fluminense, Rio de Janeiro.

[14] PALADY, Paul. FMEA análise dos modos de falha e efeitos. São Paulo: IMAN, 1997.

[15] PMI. Guia do conhecimento em gerenciamento de projetos - Guia PMBOK. 6 ${ }^{\mathrm{a}}$ ed. Newton Square, Pensilvânia: PMI, 2017.

[16] REIS, Estêvão R. A. Avaliação de riscos na atividade de Manutenção. Estudo de caso. 2015. Dissertação (Mestrado em Engenharia Mecânica). Área Departamental de Engenharia Mecânica - Instituto Superior de Engenharia de Lisboa, Lisboa.

[17] SIMON, Katarina. Oil and gas storage tank risk analysis. Risk Analysis for Prevention of Hazardous Situations in Petroleum and Natural Gas Engineering. $p$. 303-321, 2014.

[18] SOUZA, Fábio H.; BARCAUI, André. Gerenciamento de riscos aplicado à contratação de serviços em projetos de manutenção na indústria petroquímica. Sistemas \& Gestão, 9(1), 142-154, 2014. Disponível em: <www.revistasg.uff.br/index.php/sg/article/viewFile/V9N1A12/SGV9N1A12> Acesso em: 20/jan/2019.

[19] WU, Yunna; LI, Lingwenying; XU, Ruhang; CHEN, Kaifeng; HU, Yong; LIN, Xiaoshan. Risk assessment in straw-based power generation public-private partnership projects in China: A fuzzy synthetic evaluation analysis. Journal of Cleaner Production, 161, p. 977-990, 2017.

[20] XU, Yelin; YEUNG, John F. Y.; CHAN, Albert P. C.; CHAN, Daniel W. M.; WANG, Shou Q.; KE, Yongjian. Developing a risk assessment model for PPP projects in China-A fuzzy synthetic evaluation approach. Automation in Construction, 19(7), $p$. 929-943, 2010. 